\title{
Dynamic Attribute-Level Best Worst Discrete Choice Experiments
}

\author{
Amanda Working ${ }^{1}$, Mohammed Alqawba ${ }^{1} \&$ Norou Diawara ${ }^{1}$ \\ ${ }^{1}$ Department of Mathematics and Statistics, Old Dominion University, Norfolk, VA, USA \\ Correspondence: Norou Diawara, Department of Mathematics and Statistics, Old Dominion University, Norfolk, \\ VA 23529, USA. E-mail: ndiawara@odu.edu
}

Received: April 12, $2019 \quad$ Accepted: May 6, $2019 \quad$ Online Published: May 21, 2019
doi:10.5539/ijms.v11n2p1
URL: https://doi.org/10.5539/ijms.v11n2p1

\begin{abstract}
Dynamic modelling of decision maker choice behavior of best and worst in discrete choice experiments (DCEs) has numerous applications. Such models are proposed under utility function of decision maker and are used in many areas including social sciences, health economics, transportation research, and health systems research. After reviewing references on the study of such experiments, we present example in DCE with emphasis on time dependent best-worst choice and discrimination between choice attributes. Numerical examples of the dynamic DCEs are simulated, and the associated expected utilities over time of the choice models are derived using Markov decision processes. The estimates are computationally consistent with decision choices over time.
\end{abstract}

Keywords: discrete choice models, best-worst scaling, Markov decision processes

\section{Introduction and Motivation}

Discrete choice experiments (DCEs) are applied in social sciences, health economics, transportation research, and health systems (see Potoglou et al., 2011; Lancsar \& Louviere, 2008; Greene \& Hensher, 2003). DCEs and their models (Discrete choice models, DCMs) focus on predicting a decision maker's choices in products or services. In many cases, they are time dependent. Such research has not been practically implemented in attribute-level best-worst DCE, in which the decision maker's task is to choose the best option and the worst option from a choice set instead of just the best option, as in traditional DCEs. This class of experiments falls under best-worst scaling (BWS) experiments (Louviere et al., 2015). Here, we apply the BWS models over a time sequence to quantify and measure decision maker behavior and derive the utilities using Markov decision processes (MDPs). The change in utilities from one time to the next is described in the form of gain or loss. The utility is composed of a systematic component that is dependent on the key attributes of the product and a random component. Train (2009) presents multiple models based on different assumptions about the distribution of the random component. In his suggested model, the error terms are assumed to be homogeneous and uncorrelated (Train, 2009). By assuming the covariates are generated under a normal distribution and the error terms under a generalized extreme value distribution, the output data is then modeled as binary and conditional logit. Our focus is on the conditional logit assumption but add a dependence structure through time and transition probabilities under MDPs.

Lancsar et al. (2013) and Louviere et al. (2015) provide three cases of the BWS experiments: 1) best-worst object scaling, 2) best-worst attribute-level scaling or profile case, and 3) best-worst discrete choice experiments (BWDCEs) or multi-profile case. We are interested in the profile case, also referred to as Case 2 (BWS). It is also of interest to us to extend the model built on a function of the data, as presented in Grasshoff et al. (2003), Grasshoff et al. (2004) and Grossmann et al. (2009), to the attribute-level best-worst DCEs. In extending this work to these experiments, we provided an additional way to define the systematic component that provides flexibility that is not seen in traditional methods.

By scaling the attributes and the attribute-levels, it is possible to determine the impact the variables have on decision maker behavior. We simulated data and computed the associated parameter estimates. The results of this simulation were used to project the expected discounted utility over time using MDPs.

The manuscript is organized as follows. In Section 2, we present the model design and properties for attribute-level best-worst experiments. Extensions of MDPs for Case 2 BWS with time dependent factor are provided in Section 3. Simulated data example of Case 2 BWS models over time and results are described in Section 4. A conclusion is provided in Section 5. 


\section{Attribute-Level Best-Worst Designs}

In traditional DCE, we have a sample of $\mathrm{n}$ decision makers with $\mathrm{J}$ alternate choices. The utility function for the ith individual selecting the $\mathrm{jth}$ choice is given as:

$$
U_{i j}=V_{i j}+\epsilon_{i j}
$$

in which $V_{i j}$ is the systematic component and $\epsilon_{i j}$ is the unobserved component, or error term, where $i=1, \ldots, n$ and $j=1, \ldots, J$.

In McFadden (1974), a common distribution for the error terms was proposed, which was the Type I extreme value distribution or Gumbel distribution. That assumption leads to the conditional logit for modelling the data. Train (2009) presented other models and associated assumptions in modelling the choice made by the decision makers. As stated in Train (2009), the most important criterion is not so much about the shape of the error terms but that the errors are correlated and possibly also the utilities. To allow for dependence in choices, the error terms may be distributed as normal, and that assumption allows the outcomes to be modelled under the probit or the generalized extreme value distribution.

Let $Y_{i j}$ denote ith subject choosing the jth alternative, in which

$$
Y_{i j}=\left\{\begin{array}{l}
1, \text { if } i^{\text {th }} \text { subject chooses the } j^{\text {th }} \text { alternative, } \\
0, \text { otherwise. }
\end{array}\right.
$$

A model that describes the behavior is described through the conditional logit, and the utility associated to the various products are then estimated, with the error term of the utility from the Type I extreme value distribution. The systematic component is given as:

$$
V_{i j}=x_{i j}^{\prime} \beta_{j},
$$

with $x_{i j}^{\prime}$ describing the ith subject's covariates on the jth alternative, and $\beta_{j}$ isdefined as the subject specific covariate estimates.

The utility is then given as in Equation (1). Hence, the probability of the jth alternative being chosen by ith subject is:

$$
P_{i j}=P\left(Y_{i j}=1\right)=\frac{\exp \left(x_{i j}^{\prime} \beta_{j}\right)}{\sum_{x_{i j^{\prime}} \in C} \exp \left(x_{i j^{\prime}}^{\prime} \beta_{j}\right)}=\frac{\exp \left(V_{i j}\right)}{\sum_{x_{i j^{\prime}} \in C} \exp \left(V_{i j^{\prime}}\right)},
$$

with $\sum_{j=1}^{J} P_{i j}=1$ and for $C$ the set of all possible choices.

The above can be seen as a special approach at the intersection of information theory (e.g., entropy function) and the multinomial logit (Anas, 1983). Such model can be enhanced by adding attributes associated with alternatives.

\subsection{Attribute-Level Best-Worst DCE}

Attribute-level best-worst scaling (or BWS) are modified DCEs designed to elicit the impact the attributes and attribute-levels have on the utility of a product. As mentioned by Louviere and Timmermans (1990), an experiment must be designed in a way to evaluate combinations of attribute-levels to obtain information about attribute impacts on utility. Best-worst attribute-level DCEs provide such an experimental design to attain these impacts.

Following the setup as described by Street and Knox (2012), there are K attributes that describe the products denoted as Ai with each attribute consisting of $l_{k}$ levels for $k=1, \ldots, K$. In the study done by Knox et al. (2012) and Knox et al. (2013) for contraceptive data, there were $K=7$ attributes, with attribute levels $l_{1}=8, l_{2}=3, l_{3}=4, l_{5}=4, l_{6}=8, l_{7}=9$, and $l_{8}=6$. One of the attributes is the contraceptive's effect on acne, and the levels associated with that attribute are no effect, improves, or worsens acne symptoms. Each product is represented by a profile $x=\left(x_{1}, \ldots, x_{K}\right)^{\prime}$ in which $x_{i}$ is the attribute level for $A_{i}$ that makes up the product where the attribute-levels take values from 1 to $l_{k}$ for $k=1, \ldots, K$. The choice task considered here is to look at the pairs of attribute-levels and build a utility function over time. For every profile, the choice set (pairs of attribute levels) is then given as:

$$
C_{x}=\left\{\left(x_{1}, x_{2}\right), \ldots,\left(x_{1}, x_{K}\right),\left(x_{2}, x_{3}\right), \ldots,\left(x_{K-1}, x_{K}\right),\left(x_{2}, x_{1}\right), \ldots,\left(x_{K}, x_{K-1}\right)\right\},
$$

where the first attribute-level is considered to be the best and the second is the worst. From the profile $C_{x}$, the decision maker evaluates the choice set and determines from the $\tau=K(K-1)$ choices given which is the 
best-worst pair.

Grasshoff and Schwabe (2015) suggested that research is needed for larger number of choice sets. Here we extended the state of choices as follows. Let there be $G$ choice sets and the associated profiles are given as,

$$
\begin{gathered}
x_{1}=\left(x_{11}, x_{12}, \ldots, x_{1 k}\right) \\
x_{2}=\left(x_{21}, x_{22}, \ldots, x_{2 k}\right) \\
\vdots \\
x_{G}=\left(x_{G 1}, x_{G 2}, \ldots, x_{G k}\right)
\end{gathered}
$$

The corresponding choice pairs for the $G$ choice sets are given in Figure 1. To simplify the notation, let $C_{x_{1}}, \ldots, C_{x_{G}}$ be denoted as $C_{1}, \ldots, C_{G}$, respectively.
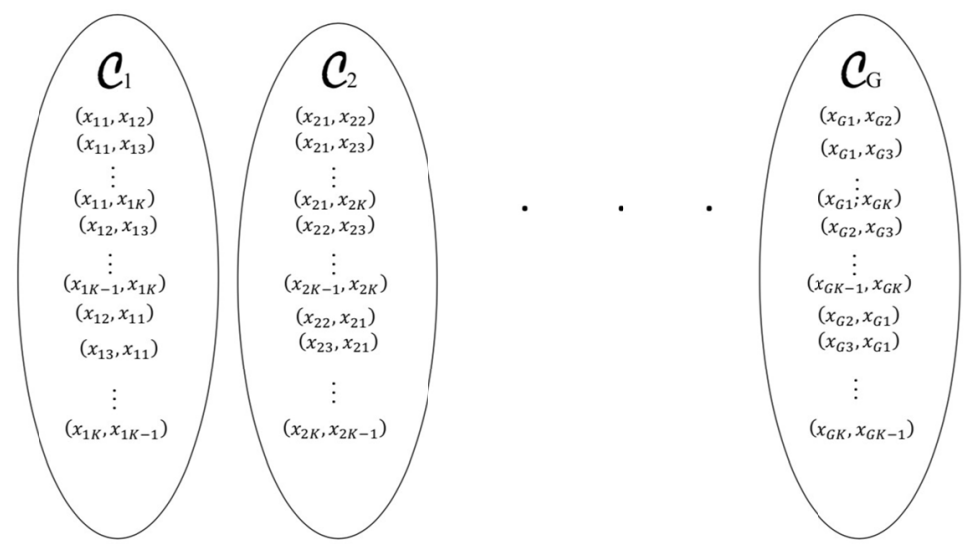

Figure 1. The $G$ choice sets in an experiment with corresponding choice pairs

Marley et al. (2008) and Street and Knox (2012) gave the best-worst choice probability for profile $x_{i}$ to be:

$$
B W_{x_{i}}\left(x_{i j}, x_{i j^{\prime}}\right)=\frac{\frac{b\left(x_{i j}\right)}{b\left(x_{i j^{\prime}}\right)}}{\sum_{\forall\left(x_{i j}, x_{i j^{\prime}}\right) \in C_{x_{i}}, j \neq j^{\prime}} \frac{b\left(x_{i j}\right)}{b\left(x_{i j^{\prime}}\right) \mid}},
$$

in which $x_{i j}$ is chosen as the best attribute-level and xij0 is the worst, and $\mathrm{b}$ is some positive scale function or impact of attribute for $j, j^{\prime}=1,2, \ldots, K, j \neq j^{\prime}$ and $i=1,2, \ldots, G$.

Thus, the following assumptions hold:

$$
B W_{x_{i}}\left(x_{i j}, x_{i j^{\prime}}\right) \geq 0, \forall i, j, \quad \text { and } \quad \sum_{\forall\left(x_{i}, x_{j}\right) \in C_{x_{i}} j \neq j^{\prime}} B W_{x_{i}}\left(x_{i j}, x_{i j^{\prime}}\right)=1 .
$$

With such assumptions, the decision maker is expected to select choices with higher BWxi values, and authors have taken advantage of selecting a subset from all possible cases.

Under random utility theory, the probability an alternative is based on the utility is defined in Equation (1). Lancsar et al. (2013) provided the utility for Case 2 BWS models and the definition of the probability as given in Equation (2) under the conditional logit model. Lipovetsky and Conklin (2014) and Marley et al. (2016) described other measures of utility of parameters as a function of $\log$ of odds. Here we consider the choice set $C_{x i}$, $\left(x_{i j}, x_{i j}{ }^{\prime}\right)$ to be the chosen pair, and the utility for choosing this pair within set $C_{x i}$ is then given by:

$$
U_{i j j^{\prime}}=V_{i j j^{\prime}}+\epsilon_{i j j^{\prime}}
$$

in which $V_{i j j^{\prime}}$ is the systematic component, $\epsilon_{i j j^{\prime}}$ is the error term, $j, j^{\prime}=1,2, \ldots, K, j \neq j^{\prime}$, and $i=1,2, \ldots, G$.

The systematic component can be expressed as,

$$
V_{i j j^{\prime}}=V_{i j}-V_{i j^{\prime}}=\left(x_{i j}-x_{i j^{\prime}}\right)^{\prime} \beta,
$$

for $x_{i j}$ and $x_{i j^{\prime}}$ as in Equation (1) and $\beta$ parameter vector of best and worst choice. The data $x_{i j}$ are indicators of the $i^{\text {th }}$ attribute $x_{i j} \in A_{j}$ and its $j^{\text {th }}$ attribute-level $x_{i j}$. The systematic component $V_{i j}$ is written as:

$$
V_{i j}=\beta_{A_{i}}+\beta_{A_{i} x_{i j}}
$$


Under the conditional logit, the probability that $\left(x_{i j}-x_{i j^{\prime}}\right)$, also denoted $x_{i j j^{\prime}}$ is chosen as

$$
P_{i j j^{\prime}}=\frac{\exp \left(V_{i j j^{\prime}}\right)}{\Sigma_{\forall\left(x_{i}, x_{j}\right) \in C_{x_{i}, j \neq j^{\prime}}} \exp \left(V_{i j j^{\prime}}\right)} .
$$

Equation (2), with the choice of the scale function $b\left(x_{i j}\right)=\exp \left(\beta_{A i}+\beta_{A i} x_{i j}\right)=\exp \left(V_{i j}\right)$ becomes Equation (4). This is easily seen by:

$$
\frac{b\left(x_{i j}\right)}{b\left(x_{i j^{\prime}}\right)}=\exp \left(V_{i j}-V_{i j^{\prime}}\right)=\exp \left(V_{i j j^{\prime}}\right) \text {. }
$$

We assume the error terms come from a Type I extreme value distribution and use the conditional logit to estimate the parameter vector:

$$
\beta^{\prime}=\left(\beta_{A_{1}}, \beta_{A_{2}}, \ldots, \beta_{A_{K}}, \beta_{A_{1} 0}, \beta_{A_{1} 1}, \ldots, \beta_{A_{1} l_{1}-1}, \ldots, \beta_{A_{K} 0}, \ldots, \beta_{A_{K} l_{K}-1}\right) .
$$

To estimate these parameters, the following identifiability condition defined on the parameters of the attribute-levels must be met:

$$
\sum_{i=1}^{l_{k}} \beta_{i}=0 \quad \text { or } \quad \beta_{l_{K}}=-\sum_{j=1}^{l_{i}-1} \beta_{j}
$$

for all $k=1,2, \ldots, K$ (Street $\&$ Burgess, 2007; Flynn et al., 2008; Grasshoff et al., 2003).

The log-likelihood for estimating the model parameters based on a random sample of $n$ decision makers is given as:

$$
l(\beta)=\sum_{s=1}^{n} \sum_{i=1}^{G} \sum_{j \neq j^{\prime}} Y_{i s j} \ln P_{i j j^{\prime}}
$$

in which the response variable representing the choices within each of the choice sets for the experiment are donated as:

$$
Y_{i s j}=\left\{\begin{array}{l}
1, \text { if } s^{\text {th }} \text { respondent chooses } j^{\text {th }} \text { alternative in the } i^{\text {th }} \text { choice set, } \\
0, \text { otherwise, }
\end{array}\right.
$$

for $i=1,2, \ldots, G, s=1,2, \ldots, n$ and $j=1,2, \ldots, \tau$.

Lancsar et al. (2017) suggested connecting models, their parameters in estimating analysis and producing measures that are related to policy and practice. We include the time feature in Case 2 BWS model structure.

\subsection{Functional Form of Attribute-Level Best-Worst Discrete Choice Model}

Van Der Pol et al. (2014) presented the systematic components of the utility defined as linear functions, quadratic functions, or as stepwise functions of the attributes. Grasshoff et al. (2013) defined the functions as regression functions of the attributes and attribute-levels in the model.

In the attribute-level best-worst DCEs, the utility of the pairs is composed of the utility corresponding to the best attribute-level and the worst attribute-level. The regression functions presented in Grasshoff et al. (2003) are applied to the attributes and attribute-levels within the respective systematic components. Let $\mathbf{f}$ be the set of regression functions for the best attribute-levels in the pairs and $\mathbf{g}$ the set of regression functions for the worst attribute-levels in the pairs. The $p \times 1$ parameter vector $\boldsymbol{\beta}$ still must satisfy the identifiability condition given in Equation (5).

Taking the systematic component defined in Equation (3), the functional systematic component for the pair $\left(x_{i j}, x_{i j^{\prime}}\right)$ is defined as:

$$
V_{i j j^{\prime}}=V_{i j}-V_{i j^{\prime}}=\left(\mathbf{f}\left(x_{i j}\right)-\mathbf{g}\left(x_{i j^{\prime}}\right)\right)^{\prime} \boldsymbol{\beta},
$$

in which $j, j^{\prime}=1,2, \ldots, K, j \neq j^{\prime}$, and $i=1,2, \ldots, G$

The probability that an alternative is chosen depends on the definition of the utility and the distribution of the error terms. Referring back to Equation (4) under the conditional logit, the probability is:

$$
B W_{x_{i}}\left(x_{i j}, x_{i j^{\prime}}\right)=\frac{\exp \left(V_{i j j^{\prime}}\right)}{\sum_{\left(x_{i q}, x_{i q^{\prime}}\right) \in C_{i}} \exp \left(V_{i q q^{\prime}}\right)}
$$




$$
\begin{aligned}
& =\frac{\exp \left(V_{i j}-V_{i j^{\prime}}\right)}{\sum_{\left(x_{i q^{\prime}}, x_{i q^{\prime}}\right) \in C_{i}} \exp \left(V_{i q}-V_{i q^{\prime}}\right)} \\
& =\frac{\exp \left(\left(\mathbf{f}\left(x_{i j}\right)-\mathbf{g}\left(x_{i j^{\prime}}\right)\right)^{\prime} \boldsymbol{\beta}\right)}{\sum_{\left(x_{i q^{\prime}}, x_{i q^{\prime}}\right) \in C_{i}} \exp \left(\left(\mathbf{f}\left(x_{i q}\right)-\mathbf{g}\left(x_{i q^{\prime}}\right)\right)^{\prime} \boldsymbol{\beta}\right)},
\end{aligned}
$$

in which $i=1,2, \ldots, G, j, j^{\prime}=1,2, \ldots, K$, and $j \neq j^{\prime}$.

In the traditional attribute-level best-worst DCE, the regression functions $f$ and $g$ are defined as indicator functions. The indicator functions are $p \times 1$ vectors. For the attributes, they are defined as,

$$
I_{A_{k}}\left(x_{i j}\right)=\left\{\begin{array}{l}
1, \text { if } x_{i j} \in A_{k}, \\
0, \text { otherwise, }
\end{array}\right.
$$

and for the attribute-levels as,

$$
I_{A_{k} x_{k}}\left(x_{i j}\right)=\left\{\begin{array}{l}
1, \text { if } x_{i j}=x_{k} \text { for } x_{i j} \in A_{k} \\
0, \quad \text { otherwise }
\end{array}\right.
$$

in which $j, k=1,2, \ldots, K$ and $i=1,2, \ldots, G$.

By rewriting the indicator functions of the $A_{k}$ and $A_{k} x_{k}$, a more general form of the regression functions can be defined. Let $b_{A k}$ and $b_{A k} x_{k}$ be constants corresponding to the best attribute and attribute-levels in a pair, and $w_{A k}$ and $w_{A k} x_{k}$ be constants corresponding to the worst attribute and attribute-levels in a pair, in which $x_{k}=1,2, \ldots, l_{k}$ and $k=1,2, \ldots, K$. The regression functions $\mathrm{f}$ and $\mathrm{g}$ are given as,

$$
\mathbf{f}\left(x_{i j}\right)=\sum_{k=1}^{K}\left[b_{A_{k}} I_{A_{k}}\left(x_{i j}\right)+\sum_{j=1}^{l_{k}} b_{A_{k x_{k}}} I_{A_{k} x_{k}}\left(x_{i j}\right)\right]
$$

and

$$
\mathbf{g}\left(x_{i j^{\prime}}\right)=-\sum_{k=1}^{K}\left[w_{A_{k}} I_{A_{k}}\left(x_{i j^{\prime}}\right)+\sum_{j=1}^{l_{k}} w_{A_{k x_{k}}} I_{A_{k} x_{k}}\left(x_{i j^{\prime}}\right)\right]
$$

in which $j, j^{\prime}=1,2, \ldots, K, j \neq j^{\prime}$, and $i=1,2, \ldots, G$.

The regression functions defined in this way provide flexibility for the traditional attribute-level best-worst DCEs. Since consumer preference for products are constantly evaluated, the data collected on a product may be dynamic. The addition of these constants to the regression functions provides researchers the ability to scale the data to reflect current trends or changes in the products. For example, let us consider that the products being modeled are pharmaceuticals such as the contraceptives proposed in Knox et al. (2012) and Knox et al. (2013). If new information about a brand of contraceptives posing a health risk was discovered, then using regression functions, it is possible to update the model to reflect this change. Assuming the change is to remove the brand, the attribute-level associated with the brand may have $b_{k x k}=w_{k x k}=0$, in which $x_{k}=1,2, \ldots, l_{k}$ and $k=1,2, \ldots, K$ represent its removal from the market. For all the pairs this attribute-level was in, the information the choice pair provides in terms of the other attributes and attribute-levels would remain intact. The model would be estimated again and the parameter vector, $\boldsymbol{\beta}$, would provide the updated impact of the attributes and attribute-levels in the experiment.

\section{Time Dependent Modelling Under Markov Decision Processes}

Markov decision processes (MDPs) are sequential decisions-making processes. MDPs seek to determine the policy or set of decision rules, under which maximum reward over time is obtained. MDPs are defined by the set $(S, R, D)$, in which $S$ is the finite set of states, $R$ the set of rewards, and $D$ the set of decisions. These processes may be discrete or continuous in time with a finite or infinite horizon, respectively. Our interest is with discrete time finite horizon MDPs, that is in which $T$ is a fixed number of time periods. The rewards (or expected rewards) are maximized by the best sequential decisions over time, making MDPs a dynamic optimization tool as used in Blanchet et al. (2016) to identify the right choices of substitution behaviors of the decision makers.

Let $s_{t} \in S$ be the states occupied at time $t, r_{t}\left(s_{t}\right)$ be the reward associated with $s_{t}$, and $d_{t}\left(r_{t}, s_{t}\right)$ be the 
decision based on the possible rewards and states at time $t$. The decision process maps the movement from one state to another over time $t$ based on rewards received and on an optimal decision set. As the decision process is Markovian, the transition probability to the next state, $s_{t+1}$ is based solely on the decision made at the current state, $s_{t}$ is $p\left(s_{t+1} \mid s_{t}\right)$, in which $t=0,1, \ldots, T$ (Puterman, 2014). There is a decision rule that governs the action the decision maker makes and rewards the results from the action. The decision of choices is made such that it maximizes their rewards. Rust (1994) and Arcidiacono and Ellickson (2011) applied MDPs to DCMs. Chades et al. (2014) applied them to solve problems in an ecological setting. As they mentioned, to suggest guidance would require running several cases. To our knowledge, such a technique has not yet been applied to decision maker choice experiments with attribute and attribute-level best-worst experiments.

For DCMs, the reward is defined by the utility function, $r\left(s_{t}, d_{t}\right)=U\left(s_{t}, d_{t}\right)$, in which $d_{t}=\delta(t)$ is the decision rule at time that maximizes the utility, and the decision rule $\delta$ is the one that maximizes the expected discount utility given as the value function.

The value function for DCMs comes from Bellman's equation and is given as:

$$
V^{t}\left(x_{t}, \epsilon_{t}\right)=\max _{d_{t} \in D} E\left(\sum_{t^{\prime}=t}^{T} \gamma^{t^{\prime}-t} U\left(x_{t^{\prime}}, d_{t^{\prime}}\right)+\epsilon\left(d_{t}^{\prime}\right) \mid x_{t}, \epsilon_{t}\right),
$$

in which the discount utility rate is given by $\gamma \in(0,1)$. The steps for determining the value function follow.

The decision rule used by a decision maker is the one under which the utility is maximized, but assuming that a person's perceived utility is impacted by time. Frederick et al. (2002) reviewed the work done on the discount utility including the decision makers' discount time factor step. The discount utility rate weights the utility a person gains from an option at some ulterior time based on their current state at time $t$ and guarantees the convergence in the infinite sum of rewards.

MDPs model the sequence of decisions based on expected rewards and transition probabilities. We defined state transition as,

$$
P\left(s_{t+1} \mid s_{t}\right)=P\left(s_{t+1}=s^{\prime} \mid s_{t}=s\right)=P_{s s^{\prime}}
$$

and the corresponding transition probability of the decision can be written as $P\left(d_{t+1} \mid d_{t}, x_{t}\right)$ with the decision dt made at time $t$ that satisfies

$$
\max _{d_{t} \in D} E\left(U\left(x_{t}, \epsilon_{t}\right)\right)
$$

for $t=1,2, \ldots, T$.

Since no closed form expression for this dynamic optimization problem is available, the value functions are computed recursively via dynamic programming under backwards recursion algorithm. First, we compute,

$$
V^{T}\left(x_{T}\right)=\sum_{d_{T} \in D} U\left(x_{T}, d_{T}\right) P\left(d_{T}\right),
$$

with $P\left(d_{T}\right)$ as the probability that $d_{T}$ was made. We denote $P\left(d_{j} \mid d_{j-1}\right)$ as the transition probability of decision $d_{j}$ given previous decision $d_{j-1}$ for $j=1, \ldots, T$. Next, we move one-time step back and compute,

$$
V^{T-1}\left(x_{T-1}, d_{T-1}\right)=U\left(x_{T-1}, d_{T-1}\right)+\sum_{d_{T} \in D} \gamma V^{T}\left(x_{T}\right) P\left(d_{T} \mid d_{T-1}\right),
$$

and another,

$$
V^{T-2}\left(x_{T-2}, d_{T-2}\right)=U\left(x_{T-2}, d_{T-2}\right)+\sum_{d_{T} \in D} \gamma V^{T-1}\left(x_{T-1}, d_{T-1}\right) P\left(d_{T-1} \mid d_{T-2}\right) .
$$

Following this pattern, we get:

$$
V^{t}\left(x_{t}, d_{t}\right)=U\left(x_{t}, d_{t}\right)+\sum_{d_{T} \in D} \gamma V^{t+1}\left(x_{t+1}, d_{t+1}\right) P\left(d_{t+1} \mid d_{t}\right),
$$

for $t=1, \ldots t-1$. For these experiments, we considered discrete time finite horizon MDPs where:

- $G$ choice sets are modeled across time of length $T$.

- $x_{t}$ are the attributes and attribute-levels corresponding to the choices in $C_{g}$, for $g=1, \ldots, G$. 
- The decision set depends on the choice set evaluated $d_{t} \in D_{i}$ in which $i=1, \ldots, G$, and $t=$ $1, \ldots, T$.

- Transition probabilities depend on a set of parameters $\theta$ that are assumed to be known or data estimable. $\theta$ is a function of an attribute and attribute level not necessarily identical to $\beta$, as described in Arcidiacono and Ellickson (2011).

- Transition probability matrices are dependent on time and on the choice set being evaluated.

There are $G$ choice sets with $\tau=K(K-1)$ choice pairs in each set. To compute the transition probabilities, the parameters are assumed known (Arcidiacono \& Ellickson, 2011). Let $\theta_{d_{t+1} \mid d_{t}}$ be the parameter vector for the transition probability from choice $d_{t}$ to $d_{t+1}$ that captures a decision maker's behavior or preference.

Let the choice pair $\left(x_{j}, x_{j^{\prime}}\right)$ denote the $q^{\text {th }}$ best-worst choice pair. Compute

$$
\pi_{q r}=\exp \left(\left(x_{j}-x_{j^{\prime}}\right)^{\prime} \theta_{q \mid r}\right) \text {, }
$$

for $j, j^{\prime}=1, \ldots, J$, where $J$ is the total possible choices for experiment, and $\theta_{q \mid r}$ are the parameters estimating the transition from choice $r$ to choice $q$.

In Case 2 BWS models, a set of $G$ choice sets are considered in the experiment. Applied MDPs, there exists a set of states $s_{t} \in S$ and possible decisions in $d_{t} \in D$ for $t=1, \ldots, T$. For Case 2 BWS MDPs, the possible states in each choice set are the alternatives, and the decision made at each time point will also be one of the alternatives. For choice set $C_{i}$ the state $s_{t i}$ and decision $d_{t i}$ are such that $1 \leq s_{t i}, d_{t i} \leq \tau$ in which $i=1, \ldots, G$ and $t=1, \ldots, T$.

Let $s_{i(t+1)}=s^{\prime}{ }_{i}$ and $s_{i t}=s_{i}$, where $s_{i}^{\prime}, s_{i} \in S_{i}$ for $i=1, \ldots, G$ and $t=1, \ldots, T$. The transition probability is denoted as

in which

$$
P_{i s s^{\prime}}^{t}=P^{t}\left(s_{i}^{\prime} \mid s_{i}, \theta_{s_{i}}^{t}\right)
$$

$$
\theta_{s_{i}}^{t}=\left(\theta_{s_{i} A_{1}}^{t}, \ldots, \theta_{s_{i} A_{K}}^{t}, \theta_{S_{i} A_{1} 1}^{t}, \ldots, \theta_{s_{i} A_{K} l_{k}}^{t}\right)
$$

is the set of parameters guiding the transition from $s_{i}$ to $s_{i}^{\prime}$, for $i=1, \ldots, G$. In Case 2 BWS models, the parameters would be the measure of relative impact/preference associated with the attributes and attribute-levels corresponding to the different choice pairs, or states, given the current state is $s_{i}$, in which $i=1, \ldots, G$. Rust (2008) and Arcidiacono and Ellickson (2011) stated that $\theta_{s_{i}}^{t}$ is an assumed known under some rationale with regards to decision maker behavior or preferences.

The parameter estimates determined by fitting the conditional logit model, as described in Section 2, produce $\hat{\beta}$ a $p=K+\sum_{k=1}^{K} l_{k}$ length vector. These parameter estimates measure the relative impact of each attribute and attribute-level in the decisions made by the decision makers. The parameters $\theta_{s_{i}}^{t}$ are the assumed impacts of the attributes and attribute-levels in the decision maker's decisions, given they currently occupy state $s_{i}$. We define these parameters as functions of the parameter estimates $\hat{\beta}$, in which there is a rate of change in the impacts over time, as follows:

$$
\begin{aligned}
\widehat{\boldsymbol{\theta}}_{s_{i}}^{t}= & \left(a_{s_{i} A_{1}}(t) \hat{\beta}_{A_{1}}, \ldots, a_{s_{i} A_{K}}(t) \hat{\beta}_{A_{K},},\right. \\
& \left.a_{s_{i} A_{1} 1}(t) \hat{\beta}_{A_{1} 1}, \ldots, a_{s_{i} A_{K} l_{k}}(t) \hat{\beta}_{A_{K} l_{K}}\right),
\end{aligned}
$$

in which $a_{i}^{\prime} s$ are the time factor change and $\hat{\beta}_{A_{k}}$ and $\hat{\beta}_{A_{k} l_{k}}$ are fixed for $i=1, \ldots, G, 1 \leq s_{i} \leq \tau, k=$ $1, \ldots, K$, and $t=1, \ldots, T$. The definition of

$$
\mathbf{a}_{s_{i}}(t)=\left(a_{s_{i} A_{1}}(t), \ldots, a_{s_{i} A_{K}}(t), a_{s_{i} A_{1} 1}(t), \ldots, a_{s_{i} A_{K} l_{k}}(t)\right)
$$

depends on the state $s_{i}$ and time $t=1, \ldots, T$. We have considered $a_{s_{i} j}(t)=a_{s_{i} j}^{t}$, in which if $\left|a_{s_{i} j}\right|<1$ the impact of the attribute or attribute-level would be lessening with time, in which $i \stackrel{=}{=} 1, \ldots, G$ and $j=1, \ldots, K$.

Also, if $a_{s_{i} A}(t) \hat{\beta}_{A}=a_{s_{i} A}^{t} \hat{\beta}_{A}>0$, then the attribute or attribute-level has a positive impact evolving at the rate at $a_{s_{i} A}^{t}$ over time for $A=A_{1}, \ldots, A_{K}, i=1, \ldots, G$, and $t=1, \ldots, T$. A static, or non-time dependent, system is considered if $a_{s_{i} A}(t) \hat{\beta}_{A}=1$, where $i=1, \ldots, G, A=A_{1}, \ldots, A_{K}$, and $t=1, \ldots, T$.

These $a_{s_{i} A}(t)$ are rates of change that guide the dynamic transition of the decision process. We can easily consider them to be non-time dependent, $a_{s_{i} A}(t)=a_{s_{i} A}$, defining the transition probabilities as stationary over time. As was mentioned earlier, there are infinite possibilities in how we define the transitions. Rust (2008) stated that when using rational observation to define the transitions, many possible choice behaviors by the decision makers are possible. Chades et al. (2014) recommended running many cases to determine the transition probabilities that will maximize the expected reward. Our definition also offers infinite possibilities in terms of 
the definition; however, we defined a rate of change to consider an evolving system. In this way, the researcher can determine what they consider feasible rates and see if the system eventually evolves to the decision they desire and how long it would take to get there.

Given $\theta_{s_{i}}^{t}$ the transition probabilities may be determined using random utility theory or inverse random utility theory in the case attribute-level best-worst models as shown in Section 2.1. Let $s_{i j j^{\prime}}^{\prime}=\left(x_{i j}, x_{i j^{\prime}}\right)$, in which $j \neq j^{\prime}, j, j^{\prime}=1, \ldots, \tau$ and $i=1, \ldots, G$. The probability that $s_{i j j^{\prime}}^{\prime}$ is the chosen state means that given $\theta_{s_{i}}^{t}$, the utility for $s_{i j j^{\prime}}^{\prime}$ is the maximum utility. The transition probability is given as,

$$
\begin{aligned}
& P^{t}\left(s_{i j j^{\prime}}^{\prime} \mid s_{i}, \boldsymbol{\theta}_{s_{i}}^{t}\right)=P^{t}\left(U_{i j j^{\prime}}^{t}>U_{i k k^{\prime}}^{t}, \forall k \neq k^{\prime} \in C_{i} \mid s_{i}, \boldsymbol{\theta}_{s_{i}}^{t}\right) \\
& =P^{t}\left(V_{i j j^{\prime}}^{t}+\epsilon_{i j j^{\prime}}^{t}>V_{i k k^{\prime}}^{t}+\epsilon_{i k k^{\prime}}^{t}, \forall k \neq k^{\prime} \in C_{i} \mid s_{i}, \boldsymbol{\theta}_{s_{i}}^{t}\right) \\
& =P^{t}\left(\epsilon_{i j j^{\prime}}^{t}<\epsilon_{i k k^{\prime}}^{t}+V_{i j j^{\prime}}^{t}-V_{i k k^{\prime}}^{t}, \forall k \neq k^{\prime} \in C_{i} \mid s_{i}, \boldsymbol{\theta}_{s_{i}}^{t}\right) \text {, }
\end{aligned}
$$

in which $j \neq j^{\prime}, j, j^{\prime}=1, \ldots, \tau, i=1, \ldots, G$ and $t=1, \ldots, T$. If we assume the random error terms are independently and identically distributed as type I extreme value distribution, the probability would then be found using the conditional logit, and is given as:

$$
\begin{aligned}
P^{t}\left(s_{i j j^{\prime}}^{\prime} \mid s_{i}, \boldsymbol{\theta}_{s_{i}}^{t}\right) & =P^{t}\left(U_{i j j^{\prime}}^{t}>U_{i k k^{\prime}}^{t}, \forall k \neq k^{\prime} \in C_{i} \mid s_{i}, \boldsymbol{\theta}_{s_{i}}^{t}\right) \\
& =\frac{\exp \left(V_{i j j^{\prime}}^{t}\right)}{\sum_{k, k^{\prime} \in C_{i}} \exp \left(V_{i k k^{\prime}}^{t}\right)} \\
& =\frac{\exp \left(\left(\mathbf{f}_{t}\left(x_{i j}\right)-\mathbf{g}_{t}\left(x_{i j^{\prime}}\right)\right)^{\prime} \boldsymbol{\theta}_{s_{i}}^{t}\right)}{\sum_{k, k^{\prime} \in C_{i}} \exp \left(\left(\mathbf{f}_{t}\left(x_{i k}\right)-\mathbf{g}_{t}\left(x_{i k^{\prime}}\right)\right)^{\prime} \boldsymbol{\theta}_{s_{i}}^{t}\right)^{\prime}}
\end{aligned}
$$

in which $j \neq j^{\prime}, k \neq k^{\prime}, j, j^{\prime}=1, \ldots, \tau, i=1, \ldots, G$, and $t=1, \ldots, T$.

The transition matrix is then a $\tau \times \tau$ matrix of the form,

$$
P_{i}^{t}=\left(\begin{array}{cccccc}
P_{i 11}^{t} & P_{i 12}^{t} & . & . & . & P_{i 1 \tau}^{t} \\
P_{i 21}^{t} & P_{i 22}^{t} & \cdot & . & \cdot & P_{i 2 \tau}^{t} \\
\cdot & \cdot & \cdot & \cdot & \cdot & \cdot \\
\cdot & \cdot & \cdot & \cdot & \cdot & \cdot \\
\cdot & \cdot & \cdot & \cdot & \cdot & \cdot \\
P_{i \tau 1}^{t} & P_{i \tau 2}^{t} & . & . & . & P_{i \tau \tau}^{t}
\end{array}\right)=\left(P_{i s s^{\prime}}^{t}\right)_{\tau \times \tau}
$$

in which $i=1, \ldots, G, s, s^{\prime}=1, \ldots, \tau$, and where $\sum_{s^{\prime}=1}^{\tau} P_{i s s^{\prime}}^{t}=1$.

The transition matrix may be either stationary or dynamic in nature. In our definition of $\theta_{s_{i}}^{t}$, this is determined by the rate $a_{s_{i} A}(t)$, in which $i=1, \ldots, G, 1 \leq j \leq p$, and $t=1, \ldots, T$. In the next section, we provide simulations under stationary and dynamic transition probabilities and make comparisons.

The decision at time $t$ as denoted by $d_{t}$ are the choice pairs and are the different states within a choice set. In Case 2 BES experiments, the $d_{t}^{i}=\left(x_{i j}, x_{i j^{\prime}}\right)$, in which $i=1, \ldots, G, j, j^{\prime}=1, \ldots, K$, and $j \neq j^{\prime}$. Then the parameter vector $\theta_{d_{t}}$ is a known vector relating the decision made at time $t$ given the decision made at time $t-1$ for $t=1, \ldots, T$. Different assumptions about the parameters $\theta_{d_{t}}$ yield different results and value functions within the experiments. However, this variability in constructing these parameters allows us to view a hypothetical future and the values they offer to decision makers.

We look at the effect of varying hyper-parameters over time to compute the transition probabilities; that is, we use the previous parameter estimates as inputs into determining $\theta_{d_{t+1}}$.

For simplicity, we will first consider stationary transition matrices. That is, $\theta_{d_{t+1}}=a_{r} \theta$ in which $a_{r}$ is independent of time and will extend to dynamic version.

In practical applications, decisions on how to act or proceed would be dictated under some expected utility. To that end, a backward recursive method is then used and a dynamic planning system with the process from its starting values/stages to its goal stage is provided. 


\section{Computations}

\subsection{Data Simulation}

In the simulated example, an empirical setup is considered. We assume $K=3$ attributes with $l_{1}=2, l_{2}=3$, and $l_{3}=$ 4 attribute-levels in an unbalanced design. There are $2 \times 3 \times 4=24$ possible profiles, or products, in this experiment. The total number of attribute-levels is $L=\sum_{i=1}^{k} l_{i}=9$, and the total number of choice pairs is $J=\sum_{k=1}^{K} l_{k}\left(L-l_{k}\right)=52$.

Louviere and Woodworth (1983), Street and Knox (2012), and Grasshoff et al. (2004) discussed the benefits in using orthogonal arrays. Generally, orthogonal experimental designs are utilized in attribute-level best-worst DCEs due to the large number of profiles in a full factorial design. There is a package in R called DoE.design that creates full factorial and orthogonal designs for a given set of attributes and attribute-levels. To obtain an orthogonal design, the oa.design function is used. For this experiment, the orthogonal design returned the full factorial design, so we used the full set of 24 profiles when simulating this data.

We simulated data for $n=300$ respondents for 24 profiles. Each choice set has $\tau=K(K-1)=6$ choices to choose from. Using the parameters given in Table 1, we simulated data in R. The data was then exported from R into the SAS ${ }^{\mathrm{R}}$ environment. Using the $\mathrm{SAS}{ }^{\mathrm{R}}$ procedure called MDC (multinomial discrete choice), the conditional logit model was fitted to the data. The parameter estimates for the generated data are given in Table 1. The parameter estimates are close to the original parameters for this example. Using the parameter estimates, the choice utilities were computed and are used to determine the expected utility/value function. The best and worst 3 choice pairs along with their utilities are presented in Table 2 and Table 3, respectively. As expected, the opposite of the pairs with the highest utilities have the lowest utilities. We Also consider an example where the model is built on the regression functions $f$ and $g$ of the data. We define $f$ and $g$ as given in Equations (10) and (11). The weights used in the regression functions are given as: $b A 1=w A 1=5, b A 2=w A 2=-2, b A 3=w A 3=1$, $b A 11=w A 11=b A 12=w A 12=5, b A 21=w A 21=b A 22=w A 22=b A 23=w A 23=-2$ and $b A 31=w A 31=b A 32$ $=w A 32=b A 33=w A 33=b A 34=w A 34=1$.

The conditional logit model is fit to the data and the resulting parameter estimates are given in Table 1 . The parameter estimates provide the adjusted attribute and attribute-level impacts.

Table 1. Parameters and parameter estimates for simulated example

\begin{tabular}{llllll}
\hline & \multicolumn{3}{c}{ Estimates } & \multicolumn{2}{c}{ Functional Form } \\
Parameters & $\beta$ & $\hat{\beta}$ & SE & $\hat{\beta}$ & SE \\
\hline$\beta A_{1}$ & -2.0000 & -2.0711 & 0.0621 & -0.3915 & 0.0289 \\
$\beta A_{2}$ & 1.5000 & 1.5248 & 0.0438 & -0.7604 & 0.0082 \\
$\beta A_{3}$ & $*$ & $*$ & $*$ & $*$ & $*$ \\
$\beta A_{1} 1$ & -2.0000 & -2.0308 & 0.0619 & -0.3935 & 0.0288 \\
$\beta A_{1} 2$ & 2.0000 & 2.0308 & $*$ & 0.3935 & $*$ \\
$\beta A_{2} 1$ & 1.9900 & 2.0970 & 0.0804 & -0.9660 & 0.0148 \\
$\beta A_{2} 2$ & -0.2900 & -0.3567 & 0.0482 & 0.1370 & 0.0092 \\
$\beta A_{2} 3$ & -1.7000 & -1.7403 & $*$ & 0.8290 & $*$ \\
$\beta A_{3} 1$ & -0.9200 & -0.8914 & 0.0407 & -0.8867 & 0.0410 \\
$\beta A_{3} 2$ & -0.1800 & -0.1805 & 0.0368 & -0.1806 & 0.0368 \\
$\beta A_{3} 3$ & 0.5000 & 0.4911 & 0.0369 & 0.4966 & 0.0366 \\
$\beta A_{3} 4$ & 0.6000 & 0.5808 & $*$ & 0.5707 & $*$ \\
\hline
\end{tabular}

Table 2. Choice pairs with the highest utility in the experiment

\begin{tabular}{lllll}
\hline Best Attribute & Level & Worst Attribute & Level & Utility \\
\hline 2 & 1 & 1 & 1 & 12.3633 \\
2 & 2 & 1 & 1 & 8.8012 \\
3 & 4 & 1 & 1 & 7.6931 \\
\hline
\end{tabular}

Table 3. Choice pairs with the lowest utility in the experiment

\begin{tabular}{lllll}
\hline Best Attribute & Level & Worst Attribute & Level & Utility \\
\hline 1 & 1 & 2 & 1 & -9.2594 \\
1 & 1 & 2 & 2 & -6.5358 \\
1 & 1 & 3 & 4 & -5.7929 \\
\hline
\end{tabular}


To build preference choices over time, we next extend the Case 2 BWS experiment of choice pairs and describing the optimal variation over $T=5$ time periods. Under that experiment, the decision maker chooses an alternative that provides maximum utility of attributes and attribute-levels over time. Numerical maximization to find the expected utility under Bellman's equation of the MDPs will be used under two cases. Under Case 1, stationary transition probabilities are considered while dynamic transition probabilities are presented under Case 2 .

\subsection{Case 1: Stationary Transition Probabilities}

We ran the simulation under this case with an advantageous proposed structure. The intent is to validate/justify our relative performance over time under stationary sparsity.

In this example, respondents are assumed to make similar decisions at each decision epoch that they made at the previous time point. The transition parameters $\boldsymbol{\theta}_{s_{i}}^{t}$ where $s_{i}^{t}=\left(x_{i j}, x_{i j^{\prime}}\right)$, are defined as for the attributes as,

$$
\boldsymbol{\theta}_{s_{i} A_{k}}^{t}=\left\{\begin{array}{cl}
1.7\left|\beta_{A_{k}}\right|, & \text { if } x_{i j} \in A_{k}, \\
-1.7\left|\beta_{A_{k}}\right|, & \text { if } x_{i j^{\prime}} \in A_{k}, \\
\beta_{A_{k}}, & \text { otherwise, }
\end{array}\right.
$$

and for the attribute-levels,

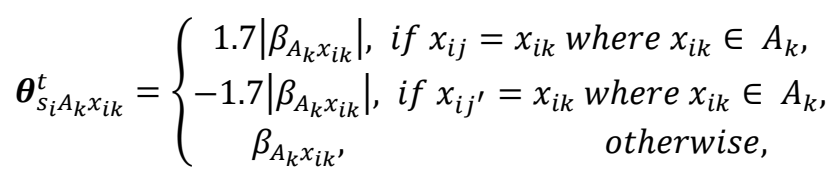

where $j \neq j^{\prime}, j, j^{\prime}, k=1,2, \ldots, K, 1 \leq x_{k} \leq l_{k}$, and $i=1,2, \ldots, G$. The transition parameters do not change with time, so the transition matrix is stationary. The goal of this case was to design the transition probabilities in a way that the choice made at $t$ is most likely to be made at $t+1$. If we considered $a_{s i} m(t)=\beta_{m}$ for $i=1,2, \ldots, G$, and $m=1,2, \ldots, p$, then the system would remain static and every row of the transition matrix would be the same. Recall that $p=K+\sum_{k=1}^{K} l_{k}=12$ is the number of parameters. We consider $1.7\left|\beta_{m}\right|$ when a state or choice pair at time $t+1$ has the same best attribute and attribute-level as the state occupied at time $t$, and $-1.7\left|\beta_{m}\right|$ when a state or choice pair at time $t+1$ has the same worst attribute and attribute-level as the state occupied at time $t$. We consider $\left|\beta_{m}\right|$ to control the direction of the impact making sure it is positive for the best attribute and attribute-level of $s_{i}$ and use $-\left|\beta_{m}\right|$ to make sure its negative for the worst attribute and attribute-level of $s_{i}$. We use 1.7 to increase the impact of the best and worst attributes and attribute-levels of $s_{i}$. The definition of $a_{s i} m(t)$ in this way insures that states with common best and worst attributes and attribute levels as the present state occupied, $s_{i}^{t}=\left(x_{i j}, x_{i j^{\prime}}\right)$, have a greater probability of being transitioned to, where $i=1,2, \ldots, G, j \neq j^{\prime}, j, j^{\prime}=1,2, \ldots, K$, and $t=1,2, \ldots, T$. The weights associated to the attributes and attribute levels are selected as above.

Referring back to Section 3, the systematic component as a function of the best and worst attribute-level in the pair, is:

where $\mathrm{f}_{t}$ and $\mathrm{g}_{t}$, are given as:

$$
V_{i j j^{\prime}}=\left(\boldsymbol{f}_{t}\left(x_{i j}\right)-\mathbf{g}_{t}\left(x_{i j^{\prime}}\right)\right)^{\prime} \beta,
$$

$$
\boldsymbol{f}_{t}\left(x_{i j}\right)=\sum_{k=1}^{K}\left[b_{A_{k}}^{t} I_{A_{k}}\left(x_{i j}\right)+\sum_{j=1}^{l_{k}} b_{A_{k} x_{k}}^{t} I_{A_{k} x_{k}}\left(x_{i j}\right)\right]
$$

and

$$
\mathbf{g}_{t}\left(x_{i j^{\prime}}\right)=-\sum_{k=1}^{K}\left[w_{A_{k}}^{t} I_{A_{k}}\left(x_{i j^{\prime}}\right)+\sum_{j=1}^{l_{k}} w_{A_{k}}^{t} x_{k} I_{A_{k} x_{k}}\left(x_{i j^{\prime}}\right)\right],
$$

where $j, j^{\prime}=1,2, \ldots, K, j \neq j^{\prime}$, and $i=1,2, \ldots, G$.

Table 4. Stationary transition matrix in Case 1 for Profile 1

\begin{tabular}{lllllll}
\hline$\left(x_{12}, X_{22}\right)$ & 0.9837 & 0.0000 & 0.0136 & 0.0000 & 0.0000 & 0.0027 \\
$\left(x_{22}, X_{12}\right)$ & 0.0000 & 0.8924 & 0.0000 & 0.1074 & 0.0003 & 0.0000 \\
$\left(x_{12}, X_{34}\right)$ & 0.0038 & 0.0000 & 0.9932 & 0.0000 & 0.0030 & 0.0000 \\
$\left(x_{34}, X_{12}\right)$ & 0.0000 & 0.0038 & 0.0000 & 0.9613 & 0.0000 & 0.0003 \\
$\left(x_{22}, X_{34}\right)$ & 0.0000 & 0.4289 & 0.0004 & 0.0002 & 0.5705 & 0.0000 \\
$\left(x_{34}, X_{22}\right)$ & 0.0001 & 0.0004 & 0.0000 & 0.7113 & 0.0000 & 0.2882 \\
\hline
\end{tabular}


The difference in the value functions over time for Profile 1 are displayed in Figure 2. Choice pair $\left(x_{22}, x_{12}\right)$, where $x_{22}$ is the $2^{\text {nd }}$ level of attribute 2 is the best and $x_{12}$ is the $2^{\text {nd }}$ level of attribute 1 is the worst, is the choice with the highest expected utility. The opposite pair $\left(x_{12}, x_{22}\right)$ is the worst choice pair. However, $\left(x_{12}, x_{22}\right)$ 's utility increases significantly between time $t=3$ and $t=4$ because of the change in the weights applied to the attributes and attribute-levels.

The model applied here views the attribute-level best-worst DCEs as sequential leading to a partial separation best-worst choices over time. Validity is guided by the transition probabilities under Case 1, the participants follow the same choice preferences. In Table 4, the transition probabilities are generally highest on the diagonal and the same at each time period as we would expect in this setup. As expected the trend in the utility is kept.

\section{Profile 1: Difference in Value Function}

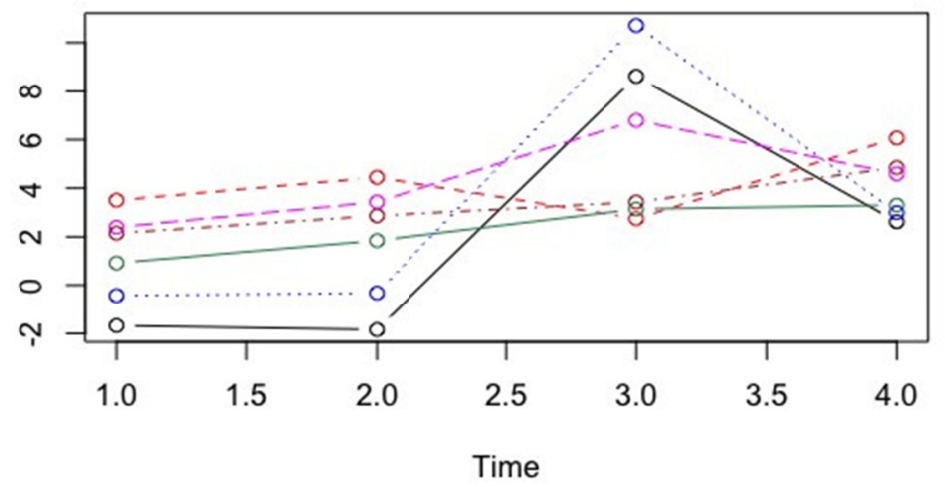

Figure 2. Expected discounted utility and their differences over time for Profile 1

\subsection{Case 2: Dynamic Transition Probabilities}

In this case, respondents are allowed to make similar decisions at each time epoch with a different rate of change, making the transition probabilities dynamic. The transition parameters $\theta_{s_{i}}^{t}$ where $s_{i}^{t}=\left(x_{i j}, x_{i j} 0\right)$ are defined as for the attributes as,

$$
\theta_{s_{i}}^{t}= \begin{cases}1.7^{t}\left|\beta_{A_{k}}\right| & \text { if } x_{i j} \in A_{k} \\ -1.7^{t}\left|\beta_{A_{k}}\right| & \text { if } x_{i j^{\prime}} \in A_{k} \\ \beta_{A_{k^{\prime}}}, & \text { otherwise, }\end{cases}
$$

and for the attribute-levels,

$$
\theta_{s_{i} A_{k} x_{i k}}^{t}=\left\{\begin{array}{c}
1.7^{t}\left|\beta_{A_{k} x_{i k} \mid}\right| \text { if } x_{i j}=x_{i k} \text { where } x_{i k} \in A_{k}, \\
-1.7^{t}\left|\beta_{A_{k} x_{i k}}\right| \text { if } x_{i j^{\prime}}=x_{i k} \text { where } x_{i k} \in A_{k}, \\
\beta_{A_{k} x_{i k}} \quad \text { otherwise, }
\end{array}\right.
$$

where $j \neq j^{\prime}, j, j^{\prime}, k=1,2, \ldots, K, 1 \leq x_{k} \leq l_{k}$, and $i=1,2, \ldots, G$.

We ran the simulation under this case with advantageous proposed hybrid structure as shown above using the functional form as described in Case 1. The transition matrix at time $t=1$ is kept the same as it was Case 1 in Table 4, and subsequent transition probabilities at time $t=2,3$, and 4 are given in Tables 5,6 , and 7, respectively. The transition probabilities are highest on the diagonal verifying the direction we wanted in the transitions. The difference in value functions for Profile 1 are displayed in Figure 3 . Choice pair $\left(x_{22}, x_{12}\right)$, where $x_{22}$ is the $2^{\text {nd }}$ level of attribute 2 is the best and $x_{12}$ is the $2^{\text {nd }}$ level of attribute 1 is the worst, still remains the choice with the highest expected utility as in Case 1 . The opposite pair $\left(x_{12}, x_{22}\right)$ is the worst choice pair. We also notice more shifts in expected utility than in previous cases for Profile 1 . Scaling the data makes the utilities shift in much more extreme values. 
Table 5. Dynamic transition matrix in Case 2 at time $t=2$ for Profile 1

\begin{tabular}{lllllll}
\hline$\left(x_{12}, X_{22}\right)$ & 0.9985 & 0.0000 & 0.0015 & 0.0000 & 0.0000 & 0.0000 \\
$\left(x_{22}, X_{12}\right)$ & 0.0000 & 0.9873 & 0.0000 & 0.0127 & 0.0000 & 0.0000 \\
$\left(x_{12}, X_{34}\right)$ & 0.0002 & 0.0000 & 0.9998 & 0.0000 & 0.0000 & 0.0000 \\
$\left(x_{34}, X_{12}\right)$ & 0.0000 & 0.0019 & 0.0000 & 0.9981 & 0.0000 & 0.0000 \\
$\left(x_{22}, X_{34}\right)$ & 0.0000 & 0.0337 & 0.0001 & 0.0000 & 0.9663 & 0.0000 \\
$\left(x_{34}, X_{22}\right)$ & 0.0000 & 0.0000 & 0.0000 & 0.2082 & 0.0000 & 0.7918 \\
\hline
\end{tabular}

Table 6. Dynamic transition matrix in Case 2 at time $t=3$ for Profile 1

\begin{tabular}{lllllll}
\hline$\left(x_{12}, x_{22}\right)$ & 1.0000 & 0.0000 & 0.0000 & 0.0000 & 0.0000 & 0.0000 \\
$\left(x_{22}, x_{12}\right)$ & 0.0000 & 0.9997 & 0.0000 & 0.0003 & 0.0000 & 0.0000 \\
$\left(x_{12}, x_{34}\right)$ & 0.0000 & 0.0000 & 1.0000 & 0.0000 & 0.0000 & 0.0000 \\
$\left(x_{34,} X_{12}\right)$ & 0.0000 & 0.0000 & 0.0000 & 1.0000 & 0.0000 & 0.0000 \\
$\left(x_{22}, X_{34}\right)$ & 0.0000 & 0.0002 & 0.0000 & 0.0000 & 0.9998 & 0.0000 \\
$\left(x_{34,} x_{22}\right)$ & 0.0000 & 0.0000 & 0.0000 & 0.0058 & 0.0000 & 0.9942 \\
\hline
\end{tabular}

Table 7. Dynamic transition matrix in Case 2 at time $t=4$ for Profile 1

\begin{tabular}{lllllll}
\hline$\left(x_{12,} X_{22}\right)$ & 1.0000 & 0.0000 & 0.0000 & 0.0000 & 0.0000 & 0.0000 \\
$\left(x_{22}, X_{12}\right)$ & 0.0000 & 1.0000 & 0.0000 & 0.0000 & 0.0000 & 0.0000 \\
$\left(x_{12}, X_{34}\right)$ & 0.0000 & 0.0000 & 1.0000 & 0.0000 & 0.0000 & 0.0000 \\
$\left(x_{34}, X_{12}\right)$ & 0.0000 & 0.0000 & 0.0000 & 1.0000 & 0.0000 & 0.0000 \\
$\left(x_{22,} X_{34}\right)$ & 0.0000 & 0.0000 & 0.0000 & 0.0000 & 1.0000 & 0.0000 \\
$\left(x_{34,} X_{22}\right)$ & 0.0000 & 0.0000 & 0.0000 & 0.0000 & 0.0000 & 1.0000 \\
\hline
\end{tabular}

Profile 1: Difference in Value Function

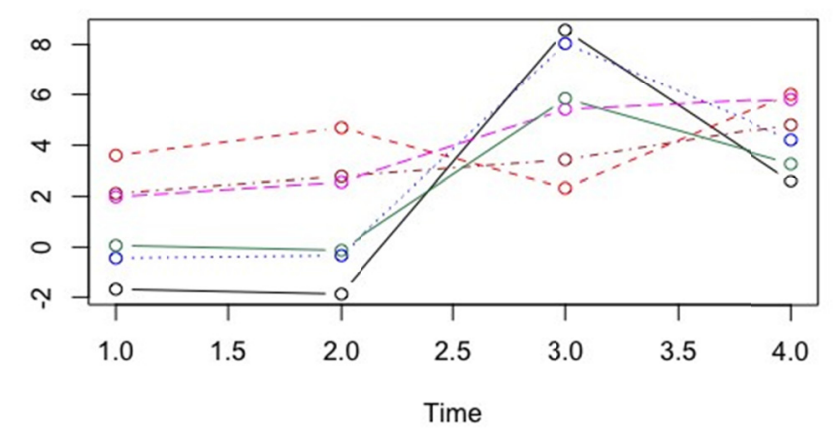

Figure 3. Expected discounted utility and their differences over time for Profile 1

\section{Conclusion}

In this paper, we have presented methodology and analysis for DCMs have applications in many areas. We adapted our simulations to match the Flynn et al. (2008) setup and extend the idea of stationarity process to a dynamic model with evaluation under random utility analysis. By encompassing the idea of choices into time dependent and with transition probabilities process, we presented a time dependent Case 2 BWS model with evaluation under random utility analysis. Our study showed that clustering can be captured, and the design can predict time stages needed to reach a target. With the simulated examples, dynamic programming algorithms reveals the highest and lowest utility trends.

A potential area of concern in the application of MDPs for attribute-level best worst DCMs is the "curse of dimensionality" as mentioned in Rust (2008). As the number of attributes, attribute-levels, and profiles grow in the experiment, the estimation process becomes exponentially more difficult. DCMs with larger number of attributes and attribute-levels have more choice sets and pairs to model across time. 


\section{Acknowledgments}

The authors are thankful to the comments and feedbacks from reviewers and editor. The latter have helped improve the quality of the manuscript. The first author also acknowledges the generous graduate assistantship support from the Virginia Modeling \& Simulation Center.

\section{References}

Anas, A. (1983). Discrete choice theory, information theory and the multinomial logit and gravity models. Transportation Research (Part B: Methodological), 17(1), 13-23. https://doi.org/10.1016/0191-2615(83)90023-1

Arcidiacono, P., \& Ellickson, P. B. (2011). Practical methods for estimation of dynamic discrete choice models. Annu. Rev. Econ., 3(1), 363-394. https://doi.org/10.1146/annurev-economics-111809-125038

Blanchet, J., Gallego, G., \& Goyal, V. (2016). A markov chain approximation to choice modeling. Operations Research, 64(4), 886-905. https://doi.org/10.1287/opre.2016.1505

Chades, I., Chapron, G., Cros, M.-J., Garcia, F., \& Sabbadin, R. (2014). Mdp toolbox: a multi-platform toolbox to solve stochastic dynamic programming problems. Ecography, 37(9), 916-920. https://doi.org/10.1111/ecog.00888

Flynn, T. N., Louviere, J. J., Peters, T. J., \& Coast, J. (2008). Best-worst scaling: what it can do for health care research and how to do it. Journal of Health Economics, 26(1), 171-189. https://doi.org/10.1186/1471-2288-8-76

Frederick, S., Loewenstein, G., \& O’Donoghue, T. (2002). Time discounting and time preference: A critical review. Journal of Economic Literature, 40(2), 351- 401. https://doi.org/10.1257/002205102320161311

Grasshoff, U., Grossmann, H., Holling, H., \& Schwabe, R. (2003). Optimal paired comparison designs for first-order interactions. Statistics, 37(5), 373-386. https://doi.org/10.1080/0233188031000154812

Grasshoff, U., Grossmann, H., Holling, H., \& Schwabe, R. (2004). Optimal designs for main effects in linear paired comparison models. Journal of Statistical Planning and Inference, 126(1), 361-376. https://doi.org/10.1016/j.jspi.2003.07.005

Grasshoff, U., Grossmann, H., Holling, H., \& Schwabe, R. (2013). Optimal design for discrete choice experiments. Journal of Statistical Planning and Inference, 143(1), 167-175. https://doi.org/10.1016/j.jspi.2012.06.019

Grasshoff, U., \& Schwabe, R. (2015). Design for discrete choice experiments. In A. Dean, M. Morris, J. Stufken \& D. Bingham, (Eds.), Handbook of design and analysis of experiment (pp. 787-826). Boca Raton, FL: Chapman and Hall/CRC.

Greene, W. H., \& Hensher, D. A. (2003). A latent class model for discrete choice analysis: contrasts with mixed logit. Transportation Research (Part B: Methodological), 37(8), 681-698. https://doi.org/10.1016/S0191-2615(02)00046-2

Grossmann, H., Grasshoff, U., \& Schwabe, R. (2009). Approximate and exact optimal designs for paired comparisons of partial profiles when there are two groups of factors. Journal of Statistical Planning and Inference, 139(3), 1171-1179. https://doi.org/10.1016/j.jspi.2008.07.006

Knox, S. A., Viney, R. C., Gu, Y., Hole, A. R., Fiebig, D. G., Street, D. J., Haas, M. R., Weisberg, E., \& Bateson, D. (2013). The effect of adverse information and positive promotion on women's preferences for prescribed $\begin{array}{lllll}\text { contraceptive products. Social Science \& } & \text { Medicine, } & \text { 83, }\end{array}$ https://doi.org/10.1016/j.socscimed.2012.12.025

Knox, S. A., Viney, R. C., Street, D. J., Haas, M. R., Fiebig, D. G., Weisberg, E., \& Bateson, D. (2012). What's good and bad about contraceptive products? Pharmacoeconomics, 30(12), 1187-1202. https://doi.org/10.2165/11598040-000000000-00000

Lancsar, E., Fiebig, D. G., \& Hole, A. R. (2017). Discrete choice experiments: A guide to model specification, estimation and software. PharmacoEconomics, 35(7), 697-716. https://doi.org/10.1007/s40273-017-0506-4

Lancsar, E., \& Louviere, J. (2008). Conducting discrete choice experiments to inform healthcare decision making. Pharmacoeconomics, 26(8), 661-677. https://doi.org/10.2165/00019053-200826080-00004

Lancsar, E., Louviere, J., Donaldson, C., Currie, G., \& Burgess, L. (2013). Best worst discrete choice experiments in health: methods and an application. Social Science \& Medicine, 76, 74-82. 
https://doi.org/10.1016/j.socscimed.2012.10.007

Lipovetsky, S., \& Conklin, M. (2014). Best-worst scaling in analytical closed form solution. Journal of Choice Modelling, 10, 60-68, https://doi.org/10.1016/j.jocm.2014.02.001

Louviere, J., \& Timmermans, H. (1990). Stated preference and choice models applied to recreation research: a review. Leisure Sciences, 12(1), 9-32, https://doi.org/10.1080/01490409009513087

Louviere, J. J., Flynn, T. N., \& Marley, A. A. J. (2015). Best-worst scaling: Theory, methods and applications. Cambridge University Press. https://doi.org/10.1017/CBO9781107337855

Louviere, J. J., \& Woodworth, G. (1983). Design and analysis of simulated consumer choice or allocation experiments: an approach based on aggregate data. Journal of Marketing Research, 20(4), 350-367. https://doi.org/10.2307/3151440

Marley, A., Flynn, T. N., \& Louviere, J. (2008). Probabilistic models of set dependent and attribute-level best-worst choice. Journal of Mathematical Psychology, 52(5), 281-296. https://doi.org/10.1016/j.jmp.2008.02.002

Marley, A., Islam, T., \& Hawkins, G. (2016). A formal and empirical comparison of two score measures for best-worst scaling. Journal of Choice Modelling, 21, 15-24. https://doi.org/10.1016/j.jocm.2016.03.002

McFadden, D. (1974). Conditional logit analysis of qualitative choice behavior. In P. Zarembka (Ed.), Frontiers in Econometrics. New York: Academic Press.

Potoglou, D., Burge, P., Flynn, T., Netten, A., Malley, J., Forder, J., \& Brazier, J. E. (2011). Best-worst scaling vs. discrete choice experiments: an empirical comparison using social care data. Social Science \& Medicine, 72(10), 1717-1727. https://doi.org/10.1016/j.socscimed.2011.03.027

Puterman, M. L. (2014). Markov decision processes: discrete stochastic dynamic programming. John Wiley \& Sons. https://doi.org/10.2307/1269932

Rust, J. (1994). Structural estimation of Markov decision processes. Handbook of Econometrics, 4, 3081-3143. https://doi.org/10.1016/S1573-4412(05)80020-0

Rust, J. (2008). Dynamic programming. In N. D. Steven \& E. B. Lawrence (Eds.), The new palgrave dictionary of economics. https://doi.org/10.1057/978-1-349-95121-5_1932-1

Street, D. J., \& Burgess, L. (2007). The construction of optimal stated choice experiments: Theory and methods (vol. 647). John Wiley \& Sons. https://doi.org/10.1002/bdm.622

Street, D. J., \& Knox, S. A. (2012). Designing for attribute-level best-worst choice experiments. Journal of Statistical Theory and Practice, 6(2), 363-375. https://doi.org/10.1080/15598608.2012.673900

Train, K. E. (2009). Discrete choice methods with simulation. Cambridge university press. https://doi.org/10.1017/CBO9780511805271

Van Der Pol, M., Currie, G., Kromm, S., \& Ryan, M. (2014). Specification of the utility function in discrete choice experiments. Value in Health, 17(2), 297-301. https://doi.org/10.1016/j.jval.2013.11.009

\section{Copyrights}

Copyright for this article is retained by the author, with first publication rights granted to the journal.

This is an open-access article distributed under the terms and conditions of the Creative Commons Attribution license (http://creativecommons.org/licenses/by/4.0/). 